\title{
E-participatory Approaches in Urban Design
}

\section{${ }^{*}{ }_{1}$ Araf Öykü Türken (D, ${ }^{2}$ Assoc. Prof. Dr Engin Eyüp Eyuboğlu}

1 Department of City and Regional Planning, Faculty of Architecture, Yildiz Technical University, Turkey

2 Department of City and Regional Planning, Faculty of Architecture, Istanbul Technical University, Turkey Email 1: araf.turken@gmail.com, Email 2: eyuboglu@itu.edu.tr

\begin{tabular}{l} 
ARTICLE INFO: \\
\hline Article History: \\
Received 9 June 2020 \\
Accepted 20 Augustus 2020 \\
Available online 8 September \\
2020 \\
\hline
\end{tabular}

Keywords:

E-participation;

Public Participation;

Urban Design.

\section{ABSTRACT}

The phenomenon of planning involving citizen's participation in planning literature has been from the second half of the 20th century. Indeed, different methods and techniques have been used in the process. However, participatory practices are time-consuming and negotiations are tiresome. Accordingly, the integration of developing digital technologies into participatory processes has been seen as a potential to reach large audiences and provide time-space independence. Within the scope of this research, a detailed literature review was done regarding e-participation, and ten (10) examples representing the upper levels at the ladder of participation were examined within the context of the project, participation, and socio-technical criteria. SWOT analyzes were structured by grouping similar applications, and current trends for the use of e-participation in urban design have been revealed. The analysis showed that citizens e participation-participation tend to allow citizen design or location-based interaction, playful interfaces and game elements which can be sources for encouragement.

JOURNAL OF CONTEMPORARY URBAN AFFAIRS (2021), 5(2), 169-182. https://doi.org/10.25034/ijcua.2021.v5n2-2

www.ijcua.com

Copyright @ 2020 Araf Öykü Türken, Engin Eyüp Eyuboğlu.

This article is published with open access at www.ijcua.com

\section{Introduction}

Participatory planning/design practices have become increasingly widespread since the second half of the 20th century and have begun to replace top-down practices. These approaches, which focus on the interaction between actors, have become stronger with concepts such as the right to the city, civic participation, and citizen power. Since the 90s, the use of digital technologies in the world and the emergence of systems such as ICT and GIS have undergone a radical change in the production process of the urban space. The forms of communication in daily life have changed, data production has reached maximum levels, and the traditional participation processes has become timeconsuming and costly. This situation required the integration of participatory planning with digital technologies. In its simplest terms, the concept of e-participation refers to the use of ICT in participatory processes. Accordingly, the ladder of participation was redefined, participatory planning met with digital

*Corresponding Author:

Department of City and Regional Planning, Faculty of Architecture, Yildiz Technical University, Istanbul, Turkey

Email address: araf.turken@gmail.com 
methods and different specialities (such as IT experts, developers) were included in the inclusive design practices. In the focus of planning and urban design, various approaches have been developed that target active participation of citizens, such as systems that allow citizen design in three-dimensional models, civic engagement platforms and participatory planning apps, co-design apps amongst others. These systems are generally designed as web-based or mobile applications. They have multiple digital methods and have goals such as collecting data by addressing large audiences, motivating participation using game elements or playful interfaces, making services transparent, creating dialogue, and increasing interaction between actors. In this context, this research examines the impact of eparticipation on urban design and planning processes and aims to understand current trends and approaches. In doing so, it adopted extensive literature research and detailed reviews of 10 international examples.

\section{Methodology}

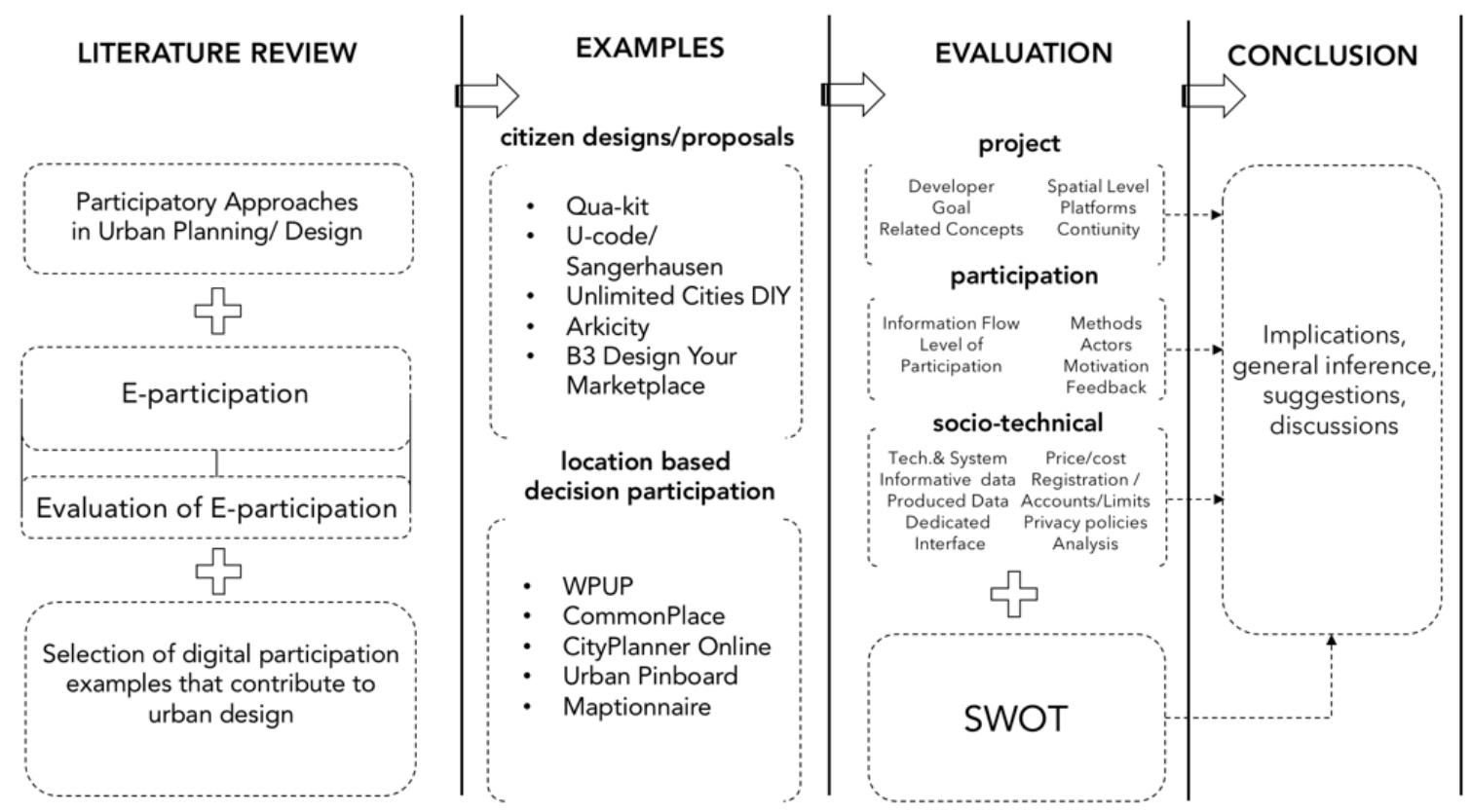

Figure 1. Structure of the Study.

Within the scope of the research, the development of participation in urban design and planning will be examined in historical order. A comprehensive literature review was conducted on e-participation, mparticipation and the use of digital tools in the participation processes. Co-design and civic engagement platforms using digital tools are searched, and the relationship with innovative city concepts such as sustainable, smart, and responsive is examined. Advanced examples focused on the spatial design on a range of street, neighborhood, and public space rather than strategic approaches, transportation was selected, and comparative studies were made on the 10 examples (Figure 1). While choosing digital participation platforms and mobile applications that contribute to urban design processes, study preference was the high levels of the participation ladder and as a system developed based on geographical or spatial data. Accordingly, in the first examples examined, citizens can visualize their ideas about the urban space (2D or 3D), while in others, citizens report decisions and suggestions for projects to be developed through urban models and online mapping. While detailing the cases, different researcher's evaluation criteria for digital participation and mobile platforms were examined, common points were determined and review parameters were structured in line with the inferences. Subsequently, 10 examples were examined in the context of the project, participation, and sociotechnical criteria. Comparisons were made on parameters such as developers, goals, spatial levels, continuity, information flow, methods, actors, motivation, technologies, 
data, price, privacy policies and analysis. Afterwards, SWOT analysis was done by grouping samples with similarities. In line with the data obtained, innovative trends and methods regarding the use of digital tools for community participation in urban design were introduced.

\section{Conceptual Framework of Participation in Urban Design/Planning}

Participation is often associated with the concept of democracy and it has a multidisciplinary, inclusive nature. In the 1930s, the Chicago School carried out field projects in disadvantaged neighborhoods, and citizen involvement was mentioned for the first time in the context of architecture and planning (Janowitz, 2015). In the 1960s, top-down transportation and transformation projects implemented in the USA increased the inequality in urban space. During this period, bottom-up approaches emerged and became widespread. In 1967, the concept of "right to the city" was introduced by Henry Lefebvre, and it was stated that only groups and societies that could take revolutionary initiative could solve urban problems (Lefebvre, 2016). Afterwards, pluralist planning approaches have replaced top-down practices; participation has been seen as a collaborative process beyond 'information'. In 1969, Arnstein published an article entitled "The Ladder of Participation", classifying participation at eight different levels (Arnstein, 1969). Simultaneously, under the principle of pluralism, planning models such as transactive, communicative and advocacy have brought a new perspective to urban planning (Table 1) differently from the rational comprehensive approach (Lane, 2005). These models targeted local mobilization and emphasized the public's role in planning and design.

Table 1. Planning Approaches and Relation with Public Participation (Arnstein, 1969; Friedmann, 1987; Hall, 1992 as cited in Lane, 2005).

\begin{tabular}{|c|c|c|c|}
\hline Level of Participation & Planning Tradition & Planning School & Planning Models \\
\hline $\begin{array}{l}\text { Citizen Control } \\
\text { Delegated Power } \\
\text { Partnership }\end{array}$ & Societal transformation & Pluralism & $\begin{array}{l}\text { Communicative } \\
\text { Bargaining } \\
\text { Marxist } \\
\text { Advocacy } \\
\text { Transactive }\end{array}$ \\
\hline $\begin{array}{l}\text { Placation } \\
\text { Consultation } \\
\text { Informing }\end{array}$ & Societal guidance & Synoptic & $\begin{array}{l}\text { Mixed scanning } \\
\text { Incrementalism } \\
\text { Synoptic planning }\end{array}$ \\
\hline $\begin{array}{l}\text { Therapy } \\
\text { Manipulation }\end{array}$ & Societal guidance & Blueprint & $\begin{array}{l}\text { Blueprint planning, } \\
\text { Geddes, Howard } \\
\text { Precinct planners }\end{array}$ \\
\hline
\end{tabular}

Thereafter, Arnstein's participation ladder was criticized as a one-way system that always aimed to reach higher levels and was reinterpreted by different professionals. In 1998, Davidson developed an approach called the "The Wheel of Participation" which has four main categories: inform consult, participate and empower. Later on, OECD (2001) established an active participation framework and categorized it by information flow directions and level of empowerment and the IAP2-Spectrum of Public Participation (2007) published an internationally accepted table emphasizing that participation levels are related to factors such as goal, promise and techniques (Commons, 2011). With the integration of digital technologies into participatory processes, different participation ladders have emerged that consider the new requirements. Although details about eparticipation are critically examined in this study, it is a point of fact that the participation processes have transformed with social needs and planning dynamics.

\section{Integration of Digital Tools into Participatory Processes}

The development of ICT has inevitably changed daily life habits, created new public spaces and redefined virtual interactive environments. In its most basic sense, digital forms of communication have great potential to eliminate communicative barriers between people and increase their networking capacity. parallel to this, the way of communication between institutions and people evolved in this new direction and created concepts such as e-democracy, egovernance, and e-participation. Macintosh (2004) expresses e-democracy as the use of ICT to support decision-making processes; he defines e-voting and e-participation as sub- 
layers of e-democracy. Accordingly, it will be useful to interpret digital technologies that affect planning and design processes.

Although the development process of computer technologies started in the 1960s, mathematical approaches in this period were insufficient to solve complex problems for the city. In the 1980s and 1990s, due to the developing GIS and other technologies, more comprehensive approaches have been developed that can provide solutions to problems related to planning and design, including topics such as data collection, data processing, visualization, analysis and project management. Following this, developments such as planning support systems and decision support systems that give priority to professional use have emerged (Klosterman, 2012). With the development of Web 2.0, content production of citizens became widespread and collaborative use of the network increased. Besides, web-based and online GIS systems have also been developed. These systems have created the PPGIS formulation integrated with the idea of community participation. Contrary to the fact that the systems in previous years were professionally oriented, these systems have great potential to ensure civic engagement and interaction between actors. It is seen that with every developing new technology, e-participation processes are evolving.

Table 2. Ladder of E-participation Through Different Perspectives.

\begin{tabular}{|c|c|c|c|c|c|}
\hline (Carver, 2001) & (Kingston, 2002) & $\begin{array}{c}\text { (Hudson-Smith, } \\
\text { Evans, Batty, \& } \\
\text { Batty, 2002) }\end{array}$ & $\begin{array}{l}\text { (Macintosh, } \\
\text { 2004) }\end{array}$ & \multicolumn{2}{|c|}{ (Krabina, 2016) } \\
\hline $\begin{array}{l}\text { Online Decision } \\
\text { Sup. Sys. }\end{array}$ & $\begin{array}{l}\text { Online Decision } \\
\text { Making }\end{array}$ & Virtual Worlds & $\begin{array}{c}\mathrm{e}- \\
\text { Empowering }\end{array}$ & Impact & \\
\hline $\begin{array}{c}\text { Online Opinion } \\
\text { Surveys }\end{array}$ & Online PPGIS & $\begin{array}{l}\text { Virtual Design } \\
\text { Studio }\end{array}$ & e-Engaging & Effective & implementation \\
\hline Online Discussion & $\begin{array}{c}\text { Online Comments } \\
\text { on App. }\end{array}$ & $\begin{array}{c}\text { Community Design } \\
\text { Sys. }\end{array}$ & \multirow[t]{7}{*}{ e-Enabling } & Intended & goal/agenda \\
\hline $\begin{array}{c}\text { Communication } \\
\text { barrier }\end{array}$ & $\begin{array}{c}\text { Online Service } \\
\text { Delivery }\end{array}$ & $\begin{array}{l}\text { Online Decision } \\
\text { Support Systems }\end{array}$ & & Active & dedicated interface \\
\hline \multirow[t]{5}{*}{$\begin{array}{l}\text { Online Service } \\
\text { Delivery }\end{array}$} & $\begin{array}{l}\text { Online Discussion } \\
\text { Forums }\end{array}$ & $\begin{array}{l}\text { Online Opinion } \\
\text { Surveys }\end{array}$ & & Implicit & awareness/connection \\
\hline & $\begin{array}{c}\text { Communication } \\
\text { barrier }\end{array}$ & Online Discussions & & \multicolumn{2}{|c|}{ Non-interaction } \\
\hline & $\begin{array}{c}\text { Online Opinion } \\
\text { Surveys }\end{array}$ & $\begin{array}{c}\text { Communication } \\
\text { barrier }\end{array}$ & & Passive & action \\
\hline & Basic Website & Online Service & & Indifference & caring/opinion \\
\hline & & Delivery & & Unawareness & information \\
\hline
\end{tabular}

One of the main parameters used when examining e-participation processes is the ladder of e-participation and e-democracy. As with Arnstein's ladder, e-participation levels increase depending on citizen empowerment. Besides, the information flow direction and the technology adopted in e-participation processes are directly related to authorization. Accordingly, the e-participation ladders developed by different professionals are compared in Table 2. For example, in the system created by Carver (2001), online services are classified as one-way, and the level of participation increases as we go towards online discussions, opinion surveys, and decision support systems. On the other hand, Kingston (2002) has positioned simple websites and opinion polls in one-way information flow while describing interactive processes as discussion forums, services, comments on apps, online PPGIS, and online decision making. Subsequently, Smith and others have added advanced technologies that can contribute to the ladder (such as community design systems, virtual design studios and virtual worlds) and re-structured high levels of participation (Hudson-Smith et al., 2002). In his article published in 2004, Macintosh displayed an attitude similar to OECD's approach (information, consultation and active participation) and grouped eparticipation as enabling, consultation and empowering. Unlike other systems, in Krabina's (2016) approach, some key issues such as the user who acquires information while unconsciously browsing the internet, implicit participation of citizen, dedicated interface, continuity of participation process are integrated into the ladder.

E-participation and collaborative participation processes are not two mutually exclusive elements; on the contrary, they contain 
methods that can be used to support each other in line with needs. The reasons for the increasing preference of e-participation today can be listed as follows: addressing large audiences, ensuring time and space independence, reducing costs, and providing support for young groups to decision-making processes for urban space. Hence, aside from methods involving face-to-face interaction such as city councils, consultation groups, workshops, negotiations, interviews, city meetings which are frequently used in the participation processes, the use of methods such as forums, online surveys, podcasts, blogs, e-petitions, e-voting, gis tools, decision-making games (Kubicek, 2009, s. 177) have increased. The technology-related structure of eparticipation also made it necessary to adopt the new actor relationships to the participation processes. With the change of tools, the processes supporting dependent or independent developers (IT professionals) have suddenly become imperative for creating dedicated interfaces, managing and analysing data exchange, ensuring the sustainability of the system and reconstructing the systems. This allowed innovative ways such as application/software competitions, media and press support to be used in designing eparticipation processes (Kassen, 2018).

It was mentioned earlier that the participation processes have been transformed in line with the prevailing technology and the needs of the age. Accordingly, e-participation processes have continued to evolve with the introduction of mobile technologies and the emergence and widespread use of devices such as smartphones and tablets. In this context, mparticipation, which is a new concept, represents the latest developments in $\mathrm{e}$ participation processes, while focusing on ensuring civic engagement through specialized 'apps' (Ertio", 2013). These applications are expressed with names such as "participatory planning apps," "citizen apps," and "civic engagement apps". It takes solutions one step further for "time/space problems" than e-participation. While classifying these applications, Ertiö (2018) separates it as environmental-centric and people-centric; he went further to mention eight different categories such as information sharing, experience, trend monitoring, integrator, nudge, local network, citizen impact, public dialogue (Ertio," 2018). Parallel to these, $\mathrm{m}$ - participation can act as a catalyst by providing advantages such as those involving passer-by citizens in the process, collecting data while providing information through applications and providing opportunities for different socio-economic groups (Fathejalali, 2017).

\subsection{E-Participatory Approaches and Related Urban Concepts}

The phenomenon of participation has been an essential component of the globally accepted sustainable city concept since the 1970s. In conferences, covenants and agreements starting with the Stockholm conference and sustainable cities such as the Rio-World Summit, The Aarhus Convention, Local Agenda 21, UN Sustainable Development Goals 2030; participation was emphasized with themes such as access to environmental information, cooperation, policymaking, active citizenship. Sustainable development goals guide not only green cities but also data-driven city concepts, smart city, digital city and responsive city. Among these, literature evidence suggests conference on the concept that is seen as a 'smart city' as dominant. Smart cities consist of six basic components: smart economy, smart governance, smart citizen, smart mobility, smart environment and smart living (Giffinger et al., 2007). Gupta, Mustafa, \& Kumar (2017) define the main elements of governance in smart city as participatory decision making, public and social services, transparent governance, political strategies and perspectives. Subsequently, what a smart citizen should have is expressed with features such as the level of qualification, openmindedness, social and ethnic plurality, flexibility, creativity, democratic, participation in public life (Gupta, Mustafa, \& Kumar, 2017). In this regard, it can be said that citizens are attributed to leading roles in data products within the scope of smart cities concept. Another city model that attributes the relationship between ICT and citizen participation to the spatial organization of the city is "responsive city." The responsive city takes citizens to the "action center" and is interested in "bringing the city back to citizens" (ETHx, 2017). Contrary to sensor data, 'responsive city' focusses on the information and data voluntarily shared by citizens (ETHX, 2017). Dominant terms in the responsive city concept are citizen science, citizen design 
science and it enables non-experts to develop ideas, considering the creative participation of the crowd (ETHx, 2017).

\subsection{Evaluation Criteria for E-participatory Platforms}

One of the first studies on the creation of eparticipation evaluation criteria were presented by Macintosh \& Whyte (2008) with eparticipation activities managed by the local government were evaluated through democracy, project, and socio-technical criteria. Within the purview of contemporary processes, the production and use of eparticipation platforms have gained speed, and they have been evaluated by many professionals for different purposes and parameters. Within the scope of the research, five articles that evaluate web-based and mobile applications to ensure community participation concerning urban space have been examined in detail (Table 3), considering the parameters, common points, and classifications used by experts.

Table 3. Evaluation Criteria for Participatory Platforms from Different Perspectives

\begin{tabular}{|c|c|c|c|c|c|c|c|c|c|}
\hline \multirow{2}{*}{\multicolumn{2}{|c|}{$\begin{array}{l}\text { E-participation } \\
\text { (Desouza \& } \\
\text { Bhagwatwar, 2012) }\end{array}$}} & \multirow{2}{*}{\multicolumn{2}{|c|}{$\begin{array}{l}\text { E-participation } \\
\text { (Desouza \& } \\
\text { Bhagwatwar, 2014) }\end{array}$}} & \multirow{2}{*}{\multicolumn{2}{|c|}{$\begin{array}{l}\text { E-participation } \\
\text { (Falco \& Kleinhans, } \\
2018 \text { ) }\end{array}$}} & \multirow{2}{*}{\multicolumn{2}{|c|}{$\begin{array}{l}\text { M-participation } \\
\text { (Ho'ffken \& Streich, } \\
2013 \text { ) }\end{array}$}} & \multirow{2}{*}{\multicolumn{2}{|c|}{$\begin{array}{l}\text { M-participation } \\
\text { (Fathejalali, 2017) }\end{array}$}} \\
\hline & & & & & & & & & \\
\hline 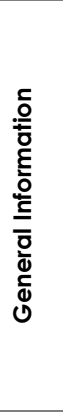 & $\begin{array}{l}\text { Name } \\
\text { Developer } \\
\text { Launch year } \\
\text { Locations } \\
\text { served } \\
\text { Platforms } \\
\text { Purpose } \\
\text { Website }\end{array}$ & 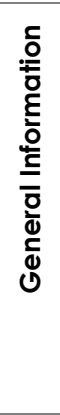 & $\begin{array}{l}\text { City } \\
\text { Name } \\
\text { Founders } \\
\text { Year } \\
\text { Goal }\end{array}$ & 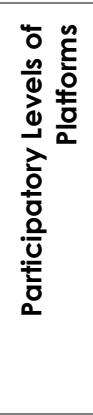 & $\begin{array}{l}\text { Self- } \\
\text { organization } \\
\text { Co- } \\
\text { production } \\
\text { Interaction } \\
\text { Consulting } \\
\text { Informing }\end{array}$ & 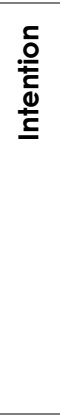 & $\begin{array}{l}\text { Name } \\
\text { Aim } \\
\text { Topic } \\
\text { Participants } \\
\text { Target group } \\
\text { Spatial } \\
\text { definition } \\
\text { Driving } \\
\text { institution }\end{array}$ & 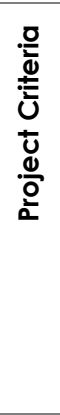 & $\begin{array}{l}\text { Name } \\
\text { Goal of } \\
\text { application } \\
\text { Beneficiaries } \\
\text { Medium } \\
\text { Topic } \\
\text { Spatial Level } \\
\text { Driving } \\
\text { institution } \\
\text { Motivation of } \\
\text { developer } \\
\text { Country }\end{array}$ \\
\hline \multirow{4}{*}{ 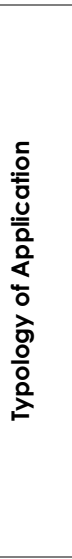 } & \multirow{4}{*}{$\begin{array}{l}\text { Transportation } \\
\text { Utilities } \\
\text { Transparency } \\
\text { and } \\
\text { corruption } \\
\text { Information \& } \\
\text { awareness \& } \\
\text { access } \\
\text { Health and } \\
\text { recreation } \\
\text { Public Safety } \\
\text { Housing }\end{array}$} & \multirow[t]{4}{*}{$\frac{n}{\frac{0}{0}}$} & $\begin{array}{l}\text { Citizen- } \\
\text { Centric\& } \\
\text { Citizen- } \\
\text { Sourced Data }\end{array}$ & \multirow{4}{*}{ 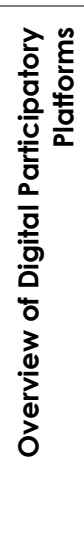 } & \multirow{4}{*}{$\begin{array}{l}\text { Name } \\
\text { Website } \\
\text { Description } \\
\text { Coverage } \\
\text { Case studies } \\
\text { Main } \\
\text { technologic } \\
\text { al } \\
\text { features } \\
\text { Pricing }\end{array}$} & \multirow{4}{*}{$\begin{array}{l}\frac{c}{0} \\
\frac{0}{0} \\
\frac{0}{0} \\
\frac{\underline{v}}{0}\end{array}$} & \multirow{4}{*}{$\begin{array}{l}\text { Approach } \\
\text { Impact (of the } \\
\text { information) } \\
\text { Ability to } \\
\text { comment } \\
\text { /data } \\
\text { Activity } \\
\text { Cost } \\
\text { Barriers to } \\
\text { registration } \\
\text { Complexity } \\
\text { Level of } \\
\text { participation }\end{array}$} & \multirow{4}{*}{ 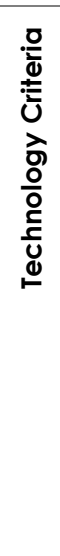 } & \multirow{4}{*}{$\begin{array}{l}\text { System of } \\
\text { project } \\
\text { Data Source } \\
\text { channel } \\
\text { Form of } \\
\text { communication } \\
\text { Platform } \\
\text { Deployed } \\
\text { Complexity } \\
\text { Location-based } \\
\text { verification. } \\
\text { Used eTools } \\
\text { Registration } \\
\text { Devices }\end{array}$} \\
\hline & & & $\begin{array}{l}\text { Citizen- } \\
\text { Centric\& } \\
\text { Gov.Open } \\
\text { Data }\end{array}$ & & & & & & \\
\hline & & & $\begin{array}{l}\text { Government- } \\
\text { Centric\&Citize } \\
\text { n-Sourced } \\
\text { Data }\end{array}$ & & & & & & \\
\hline & & & $\begin{array}{l}\text { Government- } \\
\text { Centric\&Citize } \\
\text { n-Developed } \\
\text { Solutions }\end{array}$ & & & & & & \\
\hline 웜 & $\begin{array}{l}\text { User feeds } \\
\text { Government } \\
\text { Data } \\
\text { Hybrid }\end{array}$ & \multirow{2}{*}{ 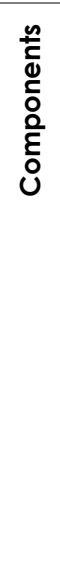 } & \multirow{2}{*}{$\begin{array}{l}\text { City } \\
\text { Platforms } \\
\text { Attractors } \\
\text { Medium } \\
\text { Information } \\
\text { and } \\
\text { knowledge } \\
\text { flows } \\
\text { Technological } \\
\text { features } \\
\text { Overall } \\
\text { framework }\end{array}$} & & & \multirow{2}{*}{ 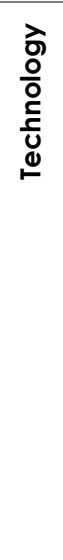 } & \multirow[t]{2}{*}{$\begin{array}{l}\text { System } \\
\text { Channels } \\
\text { App-based }\end{array}$} & \multirow{2}{*}{ 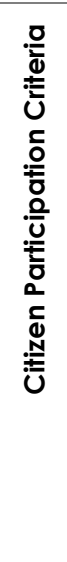 } & \multirow{2}{*}{$\begin{array}{l}\text { Involved actors } \\
\text { Level of } \\
\text { participation } \\
\text { Cost for } \\
\text { participants } \\
\text { Communication } \\
\text { direction } \\
\text { Information flow } \\
\text { Cross-media } \\
\text { communication } \\
\text { Relation } \\
\text { between actors } \\
\text { Network } \\
\text { Stage in the } \\
\text { urban planning } \\
\text { process }\end{array}$} \\
\hline 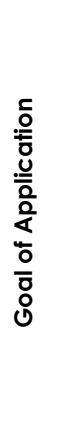 & $\begin{array}{l}\text { Opinion } \\
\text { seeking } \\
\text { Prob. } \\
\text { identificati } \\
\text { on } \\
\text { Prob. } \\
\text { resolution } \\
\text { Info, } \\
\text { access \& } \\
\text { Awareness }\end{array}$ & & & & & & & & \\
\hline
\end{tabular}




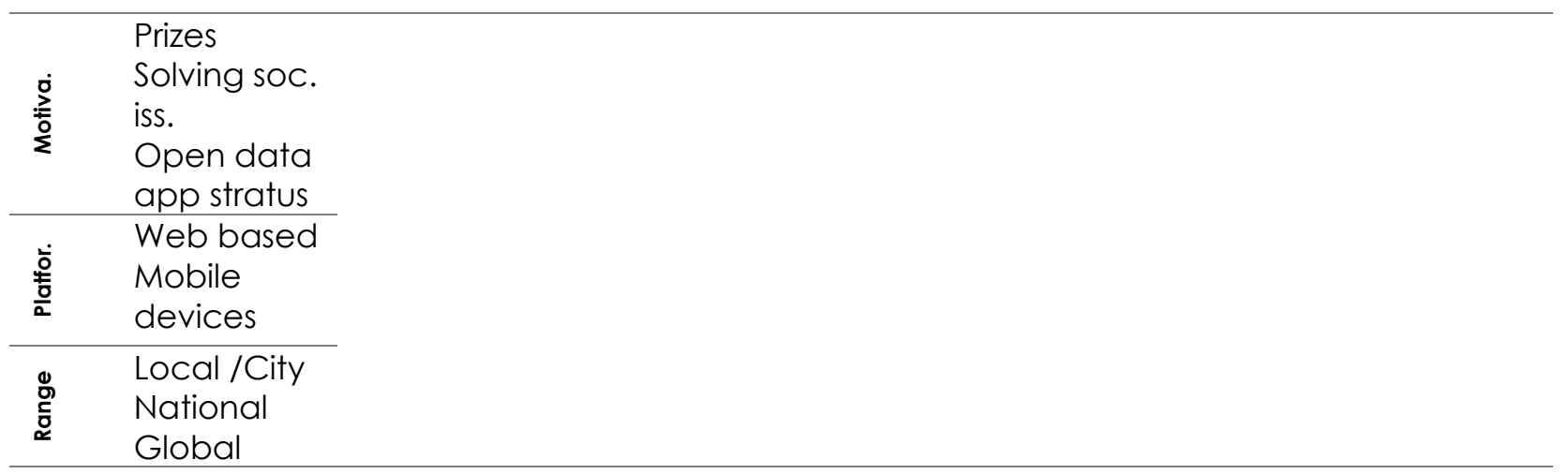

\section{Examination of Digital Participation Platforms} Ten examples contributing to community participation in urban design have been selected, and current trends and developments were examined concerning the examples. The selected cases were analysed in two groups: those that enabled the citizen to design in 2 or 3 dimensions and those that focus on making citizens' decisions or suggestions through location-based systems. The examinations are detailed under three main headings: general information about the project, parameters concerning the participatory aspect of the platforms and criteria focusing on the social and technological process. Detailed examinations of the samples can be accessed from Table 4 and Table 5 and SWOT analysis was made from the groupings.

Table 4. Examination of Platforms and Applications that Enable Citizen Design

\begin{tabular}{|c|c|c|c|c|c|c|}
\hline & & Qua-kit & $\begin{array}{l}\text { U-code / Pilot Test } \\
\text { in Sangerhausen }\end{array}$ & $\begin{array}{l}\text { B3 Design Your } \\
\text { Market Place }\end{array}$ & $\begin{array}{l}\text { Unlimited } \\
\text { Cities DIY }\end{array}$ & ArkiCity \\
\hline \multicolumn{2}{|c|}{$\begin{array}{l}\text { Main Source/ } \\
\text { References }\end{array}$} & $\begin{array}{c}\text { (Mueller \& Lu, } \\
\text { 2017) } \\
\text { (Mueller, Lu, } \\
\text { Chirkin, Klein, \& } \\
\text { Schmitt, 2018) }\end{array}$ & $\begin{array}{l}\text { (Jannack, ve } \\
\text { diğerleri, 2019) } \\
\text { (U_CODE, 2019) }\end{array}$ & $\begin{array}{c}\text { (Poplin, 2013) } \\
\text { (Geogames Lab) } \\
\text { (Thiel, 2017) }\end{array}$ & $\begin{array}{c}\text { (Hasler, } \\
\text { Chenal, \& } \\
\text { Soutter, 2017) } \\
\text { (World Urban } \\
\text { Campaign, } \\
\text { 2016) } \\
\text { (Unlimited } \\
\text { Cities DIY, } \\
\text { 2017) }\end{array}$ & (Arki_lab, 2014) \\
\hline \multirow{5}{*}{ 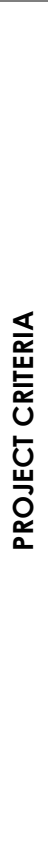 } & Developer & $\begin{array}{c}\text { ETH Zurich } \\
\text { Information Arc. } \\
\text { Artem Chirkin }\end{array}$ & $\begin{array}{c}\text { U_CODE } \\
\text { EU Horizon } 2020 \\
\text { Research and } \\
\text { Innovation Prog. }\end{array}$ & $\begin{array}{l}\text { (Student Project) } \\
\text { HafenCity and } \\
\text { (HCU) Florida } \\
\text { Atlantic University }\end{array}$ & $\begin{array}{l}\text { HOST Lab. } \\
\text { UFO (NGO) }\end{array}$ & $\begin{array}{l}\text { Arki_lab } \\
\text { Smart Inf. } \\
\text { Facilities } \\
\text { University of } \\
\text { Wollongong }\end{array}$ \\
\hline & $\begin{array}{l}\text { Goal of } \\
\text { Application }\end{array}$ & $\begin{array}{l}\text { Crowd-creative } \\
\text { participation } \\
\text { (non-experts) on } \\
\text { different urban } \\
\text { scales, by } \\
\text { arranging } \\
\text { geometries. }\end{array}$ & $\begin{array}{l}\text { A co-design } \\
\text { platform for urban } \\
\text { design allows } \\
\text { participation. }\end{array}$ & $\begin{array}{l}\text { Creating a serious } \\
\text { digital game that } \\
\text { supports playful } \\
\text { learning through a } \\
\text { real-world. }\end{array}$ & $\begin{array}{l}\text { Generate a } \\
\text { new photo- } \\
\text { realistic } \\
\text { image/collag } \\
\text { es of urban } \\
\text { space by } \\
\text { playing with } \\
\text { various } \\
\text { objects. }\end{array}$ & $\begin{array}{c}\text { Transformation } \\
\text { of urban space } \\
\text { by taking a } \\
\text { picture, making } \\
\text { a collage and } \\
\text { share online. }\end{array}$ \\
\hline & $\begin{array}{l}\text { Related } \\
\text { Concepts }\end{array}$ & $\begin{array}{c}\text { Responsive City } \\
\text { Citizen Design } \\
\text { Science }\end{array}$ & $\begin{array}{c}\text { Smart City } \\
\text { Smart Design }\end{array}$ & $\begin{array}{l}\text { Gamification in } \\
\text { Urban Planning }\end{array}$ & $\begin{array}{l}\text { Sustainable } \\
\text { City } \\
\text { Collaborative } \\
\text { Urbanism }\end{array}$ & Smart City \\
\hline & Spatial Level & $\begin{array}{c}\text { Urban Design, } \\
\text { Public Spaces } \\
\text { etc. }\end{array}$ & $\begin{array}{l}\text { Campus Design, } \\
\text { Public Spaces, } \\
\text { Urban Design etc. }\end{array}$ & $\begin{array}{l}\text { Public Spaces } \\
\text { (Market hall) }\end{array}$ & $\begin{array}{l}\text { Neighbourho } \\
\text { od, } \\
\text { public space, } \\
\text { streets etc. }\end{array}$ & $\begin{array}{l}\text { Neighbourhood, } \\
\text { public space, } \\
\text { streets etc. }\end{array}$ \\
\hline & Platforms & Web-Based & $\begin{array}{l}\text { Web-Based + } \\
\text { Mobile Devices }\end{array}$ & Web-Based & $\begin{array}{c}\text { Web-Based + } \\
\text { Mobile } \\
\text { Devices }\end{array}$ & $\begin{array}{c}\text { Mobile } \\
\text { Application }\end{array}$ \\
\hline
\end{tabular}


JOURNAL OF CONTEMPORARY URBAN AFFAIRS, 5(2), 169-182/ 2021

\begin{tabular}{|c|c|c|c|c|c|c|}
\hline & $\begin{array}{l}\text { Continuity } \\
\text { (Cases) }\end{array}$ & Yes & Pilot Test & Prototype & Yes & $\begin{array}{c}\text { Beta Version } \\
\text { Yes }\end{array}$ \\
\hline \multirow{6}{*}{ 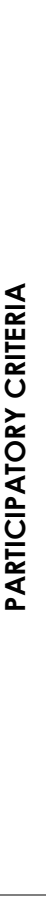 } & $\begin{array}{l}\text { Information } \\
\text { Flow }\end{array}$ & Two-way & Two-way & Two-way & Two-way & Two-way \\
\hline & $\begin{array}{l}\text { Level of } \\
\text { Participation }\end{array}$ & $\begin{array}{l}\text { High } \\
\text { Making decisions } \\
\text { online }\end{array}$ & $\begin{array}{l}\text { High (Co-design) } \\
\text { Making decisions } \\
\text { online }\end{array}$ & $\begin{array}{l}\text { High } \\
\text { Making decisions } \\
\text { online }\end{array}$ & $\begin{array}{l}\text { High (Co- } \\
\text { design) } \\
\text { Making } \\
\text { decisions } \\
\text { online } \\
\end{array}$ & $\begin{array}{c}\text { High (Co- } \\
\text { design) } \\
\text { Making } \\
\text { decisions online }\end{array}$ \\
\hline & $\begin{array}{l}\text { Methods of } \\
\text { Participation }\end{array}$ & $\begin{array}{l}\text { Community } \\
\text { design (online), } \\
\text { e-voting, add } \\
\text { comments }\end{array}$ & $\begin{array}{c}\text { Community design } \\
\text { (online), } \\
\text { touchtables, VR } \\
\text { tools, } \\
\text { ranking/voting, } \\
\text { workshops }\end{array}$ & $\begin{array}{l}\text { Community design } \\
\text { (online), e-voting, } \\
\text { discussion forums } \\
\text { etc. }\end{array}$ & $\begin{array}{c}\text { Community } \\
\text { design } \\
\text { (online), e- } \\
\text { voting, add } \\
\text { data/comme } \\
\text { nts, } \\
\text { workshops }\end{array}$ & $\begin{array}{l}\text { Community } \\
\text { design (online), } \\
\text { discussion } \\
\text { forums, } \\
\text { workshops }\end{array}$ \\
\hline & Main Actors & $\begin{array}{l}\text { Professionals, } \\
\text { Stakeholders, } \\
\text { Lab. Universities, } \\
\text { Citizen. }\end{array}$ & $\begin{array}{c}\text { Initiator, Super } \\
\text { Mediator, Planning } \\
\text { Authorities, } \\
\text { Professionals, } \\
\text { Citizen }\end{array}$ & $\begin{array}{c}\text { Universities, } \\
\text { Professionals, } \\
\text { Students, Citizen }\end{array}$ & $\begin{array}{l}\text { Municipalities } \\
\text {, Urban } \\
\text { Professionals } \\
\text { and Civil } \\
\text { society }\end{array}$ & $\begin{array}{c}\text { Municipalities, } \\
\text { Professionals, } \\
\text { Universities, Labs } \\
\text { and Citizen. }\end{array}$ \\
\hline & Motivation & $\begin{array}{l}\text { Gaming aspects, } \\
\text { Playful Design }\end{array}$ & $\begin{array}{l}\text { Crowdsourcing } \\
\text { Design Gaming }\end{array}$ & $\begin{array}{l}\text { Serious Game } \\
\text { Playful Design }\end{array}$ & $\begin{array}{l}\text { Playful } \\
\text { Design }\end{array}$ & Playful Design \\
\hline & $\begin{array}{l}\text { Feedback \& } \\
\text { Communicatio } \\
\text { n Direction }\end{array}$ & $\begin{array}{c}\text { Citizen } \leftrightarrow \text { Citizen } \\
\text { Gov/Professional } \\
\mathrm{s} \leftrightarrow \text { citizen }\end{array}$ & $\begin{array}{l}\text { Citizen } \leftrightarrow \text { Citizen } \\
\text { Gov/Professionals } \\
\leftrightarrow \text { citizen }\end{array}$ & $\begin{array}{c}\text { Citizen } \leftrightarrow \text { Citizen } \\
\text { Gov/Professionals } \leftrightarrow \\
\text { citizen }\end{array}$ & $\begin{array}{c}\text { Citizen } \leftrightarrow \\
\text { citizen } \\
\text { Citizen } \leftrightarrow \\
\text { Gov } \\
\text { /Professionals }\end{array}$ & $\begin{array}{c}\text { Citizen } \leftrightarrow \text { Citizen } \\
\text { Citizen } \leftrightarrow \text { Gov } \\
\text { /Professionals }\end{array}$ \\
\hline \multirow{8}{*}{ 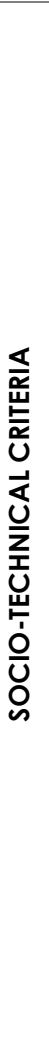 } & $\begin{array}{l}\text { Used Tech \& } \\
\text { Sys. }\end{array}$ & $\begin{array}{l}\text { Qua-kit software } \\
\text { by Artem Chirkin }\end{array}$ & $\begin{array}{l}\text { Gamification/ VR- } \\
\text { AR Applications/ } \\
\text { Crowd Analysis }\end{array}$ & $\begin{array}{l}\text { Digital Serious } \\
\text { Game Design/ } \\
\text { Adobe Flash }\end{array}$ & $\begin{array}{c}\text { Artificial } \\
\text { Intelligence } \\
\text { Analysis } \\
\text { engine } \\
\text { Automatic } \\
\text { generator }\end{array}$ & $\begin{array}{l}\text { Augmented } \\
\text { Reality } \\
\text { Mobile software } \\
\text { (for los and } \\
\text { google play) }\end{array}$ \\
\hline & $\begin{array}{l}\text { Information } \\
\text { data }\end{array}$ & $\begin{array}{l}\text { 3D typologies } \\
\text { Instructions for } \\
\text { use, Criterias, }\end{array}$ & $\begin{array}{l}\text { 3D models of } \\
\text { urban space, } \\
\text { informative data } \\
\text { etc. }\end{array}$ & $\begin{array}{l}\text { Informative data } \\
\text { through project, } \\
\text { 3D \& 2D Objects }\end{array}$ & $\begin{array}{l}\text { Project } \\
\text { packages \& } \\
\text { cutouts }\end{array}$ & $\begin{array}{c}\text { Project-specific } \\
\text { data collection } \\
\text { packages \& } \\
\text { cutouts }\end{array}$ \\
\hline & Produced Data & $\begin{array}{l}\text { Citizen Design } \\
\text { Models }\end{array}$ & $\begin{array}{l}\text { Citizen Design } \\
\text { Models }\end{array}$ & $\begin{array}{l}\text { Citizen Design } \\
\text { Models }\end{array}$ & $\begin{array}{c}\text { Citizen } \\
\text { Design } \\
\text { Images/Colla } \\
\text { ges }\end{array}$ & $\begin{array}{l}\text { Citizen Design } \\
\text { Images/Collage } \\
\text { s }\end{array}$ \\
\hline & $\begin{array}{l}\text { Dedicated } \\
\text { Interface }\end{array}$ & Yes & Yes & Yes & Yes & Yes \\
\hline & Price/cost & - & - & - & No info & No info \\
\hline & $\begin{array}{l}\text { Registration / } \\
\text { Accounts/Limits }\end{array}$ & $\begin{array}{l}\text { Professional / } \\
\text { Local Qua-kit } \\
\text { Accounts }\end{array}$ & $\begin{array}{l}\text { Tested in semi- } \\
\text { controlled with a } \\
\text { limited user. }\end{array}$ & $\begin{array}{c}\text { Tested with a limited } \\
\text { user. (students } \\
\text { \&elder) }\end{array}$ & No info & $\begin{array}{l}\text { PROJECT code is } \\
\text { required. }\end{array}$ \\
\hline & Privacy policies & No info & No info & Defined & No info & Defined \\
\hline & Analysis & $\begin{array}{c}\text { Comprehensive } \\
\text { Analysis /Form } \\
\text { and Perception } \\
\text { Based }\end{array}$ & $\begin{array}{l}\text { Comprehensive } \\
\text { Analysis/Participan } \\
\text { † \&Contribution } \\
\text { Istatis. }\end{array}$ & $\begin{array}{l}\text { User feedback and } \\
\text { ranking. }\end{array}$ & $\begin{array}{c}\text { Comprehensi } \\
\text { ve } \\
\text { Analysis } \\
\text { /artificial } \\
\text { intelligence, } \\
\text { semantic } \\
\text { analysis, } \\
\text { image } \\
\text { recognition }\end{array}$ & $\begin{array}{l}\text { Analysis /Data } \\
\text { Collection \& } \\
\text { Professionals }\end{array}$ \\
\hline
\end{tabular}

As detailed in Table 4, five different digital approaches aimed at community participation in an urban design titled Qua-kit, U-code / Sangerhausen, B3 Design Your Marketplace, Unlimited Cities DIY, ArkiCity were examined. Strengths, weaknesses, opportunities and threats within the scope of these examples are listed as follows:

Strengths: They allow users to visualize their ideas about space. The information flow is twoway and interactive. They enable the inclusion of different actors in the system and the leading roles of universities and laboratories in 
the production of the projects examined. Once the software is produced, it can be adapted to different projects and it helps to execute different participation processes with similar instructions. The sustainability of the system can be achieved in this way. Defined three-dimensional and two-dimensional objects make the system easy to understand and use. Open-source software focuses on transparency without profit. Comprehensive spatial analysis is included in most applications. Gaming and entertainment elements are used.

Weakness: The production of platforms and applications is time-consuming and costly. Expert support is required for the production of the system and adaptation to new projects. In systems with limited typology, creativity is restricted in the design process of the citizen.
Opportunities: Accessible and understandable to use. Purposeful interface design makes citizen participation enjoyable and has the potential to involve young groups in the process. Features such as authorizing the user at the point of project production, comprehensive spatial analysis capability, high level of participation, feedback systems, and open-source increases the preferability by local authorities and planning agencies.

Threats: The users may not prefer platforms whose policies of use are not defined in terms of the privacy and protection of the user's data. Applications without restrictions on registration use may cause non-local users to participate in the voting and may affect the accuracy of the data.

Table 5. Examination of Location Based Participatory Platforms.

\begin{tabular}{|c|c|c|c|c|c|c|}
\hline \multirow{2}{*}{\multicolumn{2}{|c|}{$\begin{array}{l}\text { Main Source/ } \\
\text { References }\end{array}$}} & \multirow{2}{*}{$\begin{array}{c}\text { WPUP } \\
\text { (Mansourian, } \\
\text { Taleai, \& Fasihi, } \\
2011 \text { ) } \\
\text { (Fasihi et al. } \\
\text { 2009) }\end{array}$} & \multirow{2}{*}{$\begin{array}{c}\text { Commonplace } \\
\text { (Commonplace, } \\
\text { 2013) } \\
\text { (Falco \& } \\
\text { Kleinhans, 2018) }\end{array}$} & \multirow{2}{*}{$\begin{array}{c}\text { City Planner Online } \\
\text { (CityPlannerOnline, } \\
\text { 2003) } \\
\text { (Falco \& } \\
\text { Kleinhans, 2018) }\end{array}$} & \multirow{2}{*}{$\begin{array}{c}\text { Urban Pinboard } \\
\text { (Haeusler, } \\
\text { Asher, \& Booth, } \\
2017 \text { ) }\end{array}$} & \multirow{2}{*}{$\begin{array}{l}\text { Maptionnaire } \\
\text { (Maptionnaire, } \\
2011 \text { ) (Falco \& } \\
\text { Kleinhans, 2018) }\end{array}$} \\
\hline & & & & & & \\
\hline \multirow{6}{*}{ 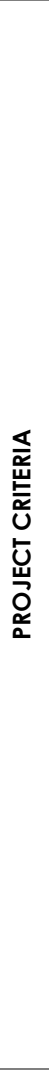 } & Developer & $\begin{array}{l}\text { K.N. Toosi } \\
\text { University of } \\
\text { Technology } \\
\text { Faculty of } \\
\text { Geodesy and } \\
\text { Geomatics } \\
\text { Engineering }\end{array}$ & $\begin{array}{l}\text { CommonPlace } \\
\text { Inc. }\end{array}$ & $\begin{array}{c}\text { Agency9 } \\
\text { Bentley Systems } \\
\text { Company }\end{array}$ & $\begin{array}{l}\text { Product of City } \\
\text { Live Labs } \\
\text { (Competition } \\
\text { Organizers: Cox } \\
\text { Arc. \& New } \\
\text { South Wales } \\
\text { University \& } \\
\text { Urban } \\
\text { Development } \\
\text { Institute of Aus- } \\
\text { tralia) } \\
\text { AAM group } \\
\text { (Geospatial } \\
\text { Services Com.) }\end{array}$ & $\begin{array}{c}\text { Maptionnaire Inc. } \\
\text { / Mapita / } \\
\text { Aalto University }\end{array}$ \\
\hline & $\begin{array}{l}\text { Goal of } \\
\text { Application }\end{array}$ & $\begin{array}{c}\text { Create } \\
\text { participatory } \\
\text { urban } \\
\text { development } \\
\text { control } \\
\text { activities for } \\
\text { land use } \\
\text { development }\end{array}$ & $\begin{array}{l}\text { Ensuring citizen } \\
\text { participation by } \\
\text { using Community } \\
\text { Heatmap and } \\
\text { Design Feedback }\end{array}$ & $\begin{array}{c}\text { Sketch, analyse, } \\
\text { and export from } \\
\text { 3D cities } \\
\text { Share/publish } \\
\text { projects and } \\
\text { crowdsource }\end{array}$ & $\begin{array}{l}\text { Platform for 3D } \\
\text { map } \\
\text { visualisation, } \\
\text { development } \\
\text { proposals \& } \\
\text { citizen } \\
\text { engagement }\end{array}$ & $\begin{array}{l}\text { Creating map- } \\
\text { surveys to get } \\
\text { idea from citizen }\end{array}$ \\
\hline & $\begin{array}{l}\text { Related } \\
\text { Concept }\end{array}$ & $\begin{array}{l}\text { Participatory } \\
\text { Planning }\end{array}$ & $\begin{array}{c}\text { Participatory } \\
\text { Planning/ Design }\end{array}$ & $\begin{array}{l}\text { Sustainable City } \\
\text { Smart City }\end{array}$ & Smart Cities & $\begin{array}{c}\text { Participatory } \\
\text { Planning/ Design }\end{array}$ \\
\hline & Spatial Level & $\begin{array}{l}\text { Urban Planning/ } \\
\text { Land-use Dec. / } \\
\text { Development } \\
\text { Control }\end{array}$ & $\begin{array}{l}\text { Neighbourhood, } \\
\text { Transportation, } \\
\text { Urban Design } \\
\text { etc. }\end{array}$ & $\begin{array}{l}\text { Architecture\& } \\
\text { Urban Design\& } \\
\text { Planning }\end{array}$ & $\begin{array}{l}\text { Architecture\& } \\
\text { Urban Design\& } \\
\text { Planning }\end{array}$ & $\begin{array}{l}\text { Urban Design \& } \\
\text { Planning }\end{array}$ \\
\hline & Platforms & Web-Based & Web-Based & Web-Based & Web-Based & Web-Based \\
\hline & $\begin{array}{l}\text { Continuity } \\
\text { (Cases) }\end{array}$ & $\begin{array}{l}\text { Prototype } \\
\text { System }\end{array}$ & Yes & Yes & $\begin{array}{c}\text { Beta Version } \\
\text { Yes }\end{array}$ & Yes \\
\hline \multirow{2}{*}{ 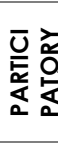 } & $\begin{array}{l}\text { Information } \\
\text { Flow }\end{array}$ & Two-way & Two-way & Two-way & Two-way & Two-way \\
\hline & $\begin{array}{l}\text { Level of } \\
\text { Participation }\end{array}$ & $\begin{array}{c}\text { High } \\
\text { Dec. Sup. Sys. }\end{array}$ & $\begin{array}{c}\text { High } \\
\text { Co-production }\end{array}$ & $\begin{array}{c}\text { High } \\
\text { Co-production }\end{array}$ & $\begin{array}{c}\text { High } \\
\text { Co-production }\end{array}$ & $\begin{array}{c}\text { High } \\
\text { Co-production }\end{array}$ \\
\hline
\end{tabular}




\begin{tabular}{|c|c|c|c|c|c|c|}
\hline & & Online PPGIS & $\begin{array}{l}\text { Map-Based } \\
\text { Consultation }\end{array}$ & $\begin{array}{c}\text { Map Based } \\
\text { Contributions + 3D } \\
\text { models }\end{array}$ & $\begin{array}{c}\text { Map Based } \\
\text { Contributions + } \\
\text { 3D models }\end{array}$ & Online PPGIS \\
\hline & $\begin{array}{l}\text { Methods of } \\
\text { Participation }\end{array}$ & $\begin{array}{l}\text { Application } \\
\text { submission, } \\
\text { discussion } \\
\text { forum. }\end{array}$ & $\begin{array}{l}\text { Online mapping, } \\
\text { surveys, e-voting, } \\
\text { discussion forums } \\
\text { etc, interviews, } \\
\text { meetings. }\end{array}$ & $\begin{array}{l}\text { Discussion forums, } \\
\text { e-voting, location- } \\
\text { based addition to } \\
\text { projects to be } \\
\text { developed }\end{array}$ & $\begin{array}{c}\text { Discussion } \\
\text { forums, e- } \\
\text { voting, } \\
\text { location-based } \\
\text { addition to } \\
\text { projects to be } \\
\text { developed }\end{array}$ & $\begin{array}{l}\text { Online mapping, } \\
\text { surveys, e-voting, } \\
\text { discussion forums } \\
\text { etc. }\end{array}$ \\
\hline & Main Actors & $\begin{array}{c}\text { Planning } \\
\text { Authorities, } \\
\text { Citizen (submit } \\
\text { and } \\
\text { participate), } \\
\text { Utility } \\
\text { Organizations }\end{array}$ & $\begin{array}{c}\text { Developers, } \\
\text { Local Authorities, } \\
\text { Citizen }\end{array}$ & $\begin{array}{l}\text { Professionals, } \\
\text { Developers, Local } \\
\text { Authorities, Citizen }\end{array}$ & $\begin{array}{c}\text { Professionals, } \\
\text { Developers, } \\
\text { Local } \\
\text { Authorities, } \\
\text { Citizen }\end{array}$ & $\begin{array}{c}\text { Municipalities, } \\
\text { Professionals, } \\
\text { Companies, } \\
\text { Agencies, Citizen }\end{array}$ \\
\hline & Motivation & $\begin{array}{c}\text { To offer } \\
\text { decisions/ } \\
\text { suggestions for } \\
\text { land use } \\
\text { development }\end{array}$ & $\begin{array}{c}\text { To offer } \\
\text { ideas/suggestions } \\
\text { for urban space }\end{array}$ & $\begin{array}{l}\text { To offer decisions/ } \\
\text { suggestions for } \\
\text { projects to be } \\
\text { developed }\end{array}$ & $\begin{array}{c}\text { To offer } \\
\text { decisions/ } \\
\text { suggestions for } \\
\text { projects to be } \\
\text { developed }\end{array}$ & $\begin{array}{c}\text { To offer } \\
\text { ideas/suggestions } \\
\text { for urban space }\end{array}$ \\
\hline & $\begin{array}{l}\text { Feedback \& } \\
\text { Communication } \\
\text { Direction }\end{array}$ & $\begin{array}{c}\text { Citizen } \leftrightarrow \text { citizen } \\
\text { Citizen } \leftrightarrow \text { Local } \\
\text { Authorities }\end{array}$ & $\begin{array}{c}\text { Citizen } \leftrightarrow \text { Gov } \\
\text { /Professionals } \\
\text { Citizen } \leftrightarrow \text { citizen }\end{array}$ & $\begin{array}{l}\text { Citizen } \leftrightarrow \text { Gov } \\
\text { /Professionals } \\
\text { Citizen } \leftrightarrow \text { citizen }\end{array}$ & $\begin{array}{l}\text { Citizen } \leftrightarrow \text { Gov } \\
\text { /Professionals } \\
\text { Citizen } \leftrightarrow \\
\text { citizen }\end{array}$ & $\begin{array}{l}\text { Citizen } \leftrightarrow \text { Gov } \\
\text { /Professionals } \\
\text { Citizen } \leftrightarrow \text { citizen } \\
\text { /depending to } \\
\text { project }\end{array}$ \\
\hline \multirow{8}{*}{ 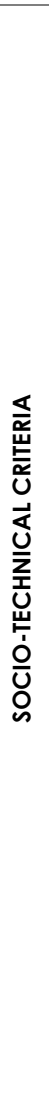 } & $\begin{array}{l}\text { Used Tech \& } \\
\text { Sys. }\end{array}$ & $\begin{array}{l}\text { Web GIS, GIS, } \\
\text { SDSS, AHP }\end{array}$ & $\begin{array}{c}\text { Software, } \\
\text { Location Based } \\
\text { Techs. }\end{array}$ & $\begin{array}{c}\text { Software, PPGIS, } \\
\text { GIS \& CAD } \\
\text { integration, WMS \& } \\
\text { Geo content }\end{array}$ & $\begin{array}{c}\text { Software, } \\
\text { WebGIS, geolT }\end{array}$ & Software, PPGIS \\
\hline & $\begin{array}{l}\text { Information } \\
\text { data }\end{array}$ & $\begin{array}{l}\text { Plan decisions, } \\
\text { spatial analysis } \\
\text { and data, } \\
\text { evaluation } \\
\text { parameters }\end{array}$ & $\begin{array}{l}\text { Maps, project } \\
\text { images, } \\
\text { information, } \\
\text { notifications }\end{array}$ & $\begin{array}{l}3 d \text { project, city } \\
\text { models, images } \\
\text { and information }\end{array}$ & $\begin{array}{l}\text { 3d project, city } \\
\text { models, images } \\
\text { and } \\
\text { information }\end{array}$ & $\begin{array}{l}\text { Maps, project } \\
\text { images, } \\
\text { information, } \\
\text { notifications }\end{array}$ \\
\hline & Produced Data & $\begin{array}{l}\text { Online spatial } \\
\text { analysis maps } \\
\text { (citizen } \\
\text { specific), } \\
\text { synthesis of the } \\
\text { participants' } \\
\text { data, opinion } \\
\text { statement. }\end{array}$ & $\begin{array}{l}\text { Citizen input to } \\
\text { urban problems } \\
\text { (report, } \\
\text { suggestions, } \\
\text { decisions on } \\
\text { projects to be } \\
\text { developed) }\end{array}$ & $\begin{array}{c}\text { Citizen input: } \\
\text { decisions, } \\
\text { comment, vote } \\
\text { Professional input: } \\
\text { models, } \\
\text { informative data } \\
\text { etc. }\end{array}$ & $\begin{array}{c}\text { Citizen input: } \\
\text { decisions, } \\
\text { comment, vote } \\
\text { Professional } \\
\text { input: models, } \\
\text { informative } \\
\text { data etc. }\end{array}$ & $\begin{array}{l}\text { Citizen input to } \\
\text { urban problems } \\
\text { (report, } \\
\text { suggestions, } \\
\text { decisions on } \\
\text { projects to be } \\
\text { development) } \\
\text { /depending } \\
\text { project }\end{array}$ \\
\hline & $\begin{array}{l}\text { Dedicated } \\
\text { Interface }\end{array}$ & Yes & Yes & Yes & Yes & Yes \\
\hline & Price/cost & - & $\begin{array}{l}\text { Yes/ For Driving } \\
\text { Institution }\end{array}$ & $\begin{array}{l}\text { Yes/ For Driving } \\
\text { Institution }\end{array}$ & $\begin{array}{c}\text { No info } \\
\text { (Beta Version) }\end{array}$ & $\begin{array}{l}\text { Yes/ For Driving } \\
\text { Institution }\end{array}$ \\
\hline & $\begin{array}{l}\text { Registration / } \\
\text { Accounts/Limits }\end{array}$ & $\begin{array}{c}\text { Only } \\
\text { Prototype }\end{array}$ & $\begin{array}{l}\text { Depending on } \\
\text { the project }\end{array}$ & $\begin{array}{l}\text { User login with } \\
\text { user-specific } \\
\text { interfaces }\end{array}$ & $\begin{array}{l}\text { User login with } \\
\text { user-specific } \\
\text { interfaces }\end{array}$ & $\begin{array}{l}\text { Depending on } \\
\text { the project }\end{array}$ \\
\hline & Privacy policies & No info & Defined & Defined & Defined & Defined \\
\hline & Analysis & $\begin{array}{c}\text { Comprehensive } \\
\text { Analysis (Spatial } \\
\text { Analysis, } \\
\text { Analytical } \\
\text { Hierarchy } \\
\text { Process) }\end{array}$ & $\begin{array}{c}\text { Comprehensive } \\
\text { Analysis (Data } \\
\text { analysis, statistics) }\end{array}$ & $\begin{array}{l}\text { Comprehensive } \\
\text { Analysis (+Spatial } \\
\text { analysis) }\end{array}$ & $\begin{array}{c}\text { Comprehensive } \\
\text { Analysis } \\
\text { (+Spatial } \\
\text { analysis) }\end{array}$ & $\begin{array}{c}\text { Comprehensive } \\
\text { Analysis (collect, } \\
\text { analyse and } \\
\text { visualise) }\end{array}$ \\
\hline
\end{tabular}

As detailed in Table 5, five different digital platforms aimed at community participation in urban planning titled WPUP, Commonplace, City Planner Online, Urban Pinboard, Maptionnaire were examined. Strengths, weaknesses, opportunities and threats are listed as follows within the scope of these examples, which are similar in terms of locationbased data production methods and technologies and information flow aspects.

Strengths: Users can view projects that are planned to be developed on real-time maps and three-dimensional city models (in 2D or $3 D$ ). With the help of simple interfaces, they 
can share location-based data, view the comments of other citizens, vote, and participate in surveys. In systems such as Maptionnaire, there are options such as mapping and route creation. All of the systems perform comprehensive analysis and have specialized interfaces. Citizens can interact directly with developers and local authorities. Examples of Maptionnaire, Commonplace, CityPlanner Online, Urban Pinboard allows the production of many different participation projects, thereby providing a time-cost advantage.

Weakness: Platforms do not allow citizens to create their designs directly. Some of the examples are poorly integrated with mobile devices. The fact that the developer and local authorities can use the systems more comprehensively has a devastating effect on the perception of the bottom-up participation process. Production of platforms and their adaptation to projects require expertise.

Opportunities: Adaptation to different projects increases preference. Three-dimensional urban models, CAD, and Gls integration enable these platforms to be used in line with different planning needs and not necessarily only in terms of community participation.

Threats: Paid uses (for beneficiary institutions) can reduce preferability. Participation in programs with three-dimensional interfaces can turn into a secondary goal. On platforms without registration limitation, the user can feel unsafe in terms of privacy and prefer not to participate. Indeed, such platforms can be manipulated.

\section{Results}

The use of digital technologies has gradually increased to enhance public participation in urban design. Platforms with strong communicative interaction have been created by using different technologies in an integrated way. Most systems are using systems without the need for additional effort and learning from the user. When the driving institutions on the platforms are examined, it is seen that universities and the private sector play leading roles especially in terms of location-based platform development and distribution. When analysed from a general perspective, common trends in digital participation platforms can be listed as follows:

- participation and community engagement as the primary goal
- allowing citizens to make their designs(2/3D) or to report their decisions and suggestions on projects to be developed with location-based systems

- providing consultation processes through three-dimensional city models and real-time maps

- enabling interaction between citizen to citizen, citizen to professionals/ local authorities/ developers at the same time thereby providing a two-way information flow through the platforms

- designing playful and dedicated interfaces to motivate citizens and increase participation. Likewise, the use of game elements or 3D city models are other supportive approaches

- to provide citizens with data security by defining terms of use and privacy

- the flexibility of systems and adaptability to more than one project; thus, ensuring continuity in the use.

- analysing process outputs and converting them into meaningful data.

Finally, it can be stated that e-participation processes will continue to evolve with developing technologies and that it will continue to support traditional participation practices. In this regard, providing freedom of design and decision making and empowering citizens in the process will strengthen the democratic aspect of e-participation.

\section{Acknowledgements}

This research did not receive any specific grant from funding agencies in the public, commercial, or not-for-profit sectors.

\section{Conflict of interests}

The authors declare no conflict of interest.

\section{References}

Arki_lab. (2014). ArkiCity Co-Create Your City. University of Wollongong. Retrieved April 2020, from https://www.arkilab.dk/arkicity/

Arnstein, S. R. (1969). A Ladder Of Citizen Participation. Journal of the American Planning Association, $\quad 35(4), \quad 216-224$. http://dx.doi.org/10.1080/01944366908977225

Carver, S. (2001). The Future of Participatory Approaches Using Geographic Information: developing a research agenda for the 21 st Century. Journal of the Urban and Regional Information 
Systems Association , 15(1), 61-71.

CityPlannerOnline. (2003). AGENCY9. CityPlanner. Retrieved April 2020, from https://cityplanneronline.com/site/

Commonplace. (2013). An online engagement hub to win the public's hearts and minds. Retrieved from https://www.commonplace.is/platform

Commons, C. (2011). Participation Models: Citizens. Youth.

Davidson, S. (1998). Spinning the wheel of empowerment. Planning, 1262(1), 14-15.

Desouza, K. C., \& Bhagwatwar, A. (2012). Citizen Apps to Solve Complex Urban Problems. Journal of Urban Technology, 19(3), 107-136. https://doi.org/10.1080/10630732.2012.673056

Desouza, K. C., \& Bhagwatwar, A. (2014). TechnologyEnabled Participatory Platforms for Civic Engagement: The Case of U.S. Cities. Journal of Urban Technology, 21(4), 25-50. https://dx.doi.org/10.1080/10630732.2014.954898

Ertiö, T. (2013). M-participation: the emergence of participatory planning applications. City of Turku Turku Urban Research Programme: Research Briefings $\quad 6 b / 2013 . \quad$ Turku, Finland. https://www.turku.fi/sites/default/files/atoms/files/t utkimuskatsauksia_2013-6b.pdf

Ertiö, T.-P. (2018). Plan on The Move: Mobile Participation in Urban Planning. University of Turku, Finland.

ETHx. (2017). Responsive Cities. Retrieved from Edx: https://www.edx.org/

Falco, E., \& Kleinhans, R. (2018). Digital Participatory Platforms for Co- Production in Urban Development: A Systematic Review. International Journal of E-Planning Research, 7(3), 1-27. https://dx.doi.org/10.4018/IJEPR.2018070105

Fasihi, A., Mansourian, A., Farnaghi, M., \& Taleai, M. (2009). A Web Based Method for Supporting Colloboration in the Urban Spatial Decision Making. Information Technologies . Lithuania. https://dx.doi.org/10.13140/2.1.1483.8724

Fathejalali, A. (2017). Enhancing Citizen Engagement in Urban Planning Processes through Mobile Participation (mParticipation). Berlin: Technischen Universität Berlin. https://depositonce.tuberlin.de/handle/11303/6244
Geogames Lab. (n.d.). B3- Design your Marketplace. Retrieved April 2020, from https:/geogameslab.net/contact/efootprints/projects/projects/b3-design-yourmarketplace/

Giffinger, R., Fertner, C., Kramar, H., Kalasek, R., Pichler, N., \& Meijers, E. (2007). Smart Cities: Ranking of European Medium-sized Cities. Final Report. Vienna UT.

Gupta, S., Mustafa, S. Z., \& Kumar, H. (2017). Smart People for Smart Cities: A Behavioral Framework for Personality and Roles. In A. K. Kar, M. P. Gupta, \& V. Ilavarasan. Advances(eds.). Smart Cities Smarter People, Governance, and Solutions (pp. 2331). https://dx.doi.org/10.1201/9781315156040

Haeusler, M. H., Asher, R., \& Booth, L. (2017). Urban Pinboard Development of a platform to access open source data to optimise urban planning performance. in Fioravanti A; Cursi S; Elahmar S; Gargaro S; Loffreda G; Novembri G; Trento A (eds.). eCAADe, 439-448.

Hasler, S., Chenal, J., \& Soutter, M. (2017). Digital Tools as a Means to Foster Inclusive, Data-informed Urban Planning. Civil Engineering and Architecture, 5(6), 230-239. https://dx.doi.org/10.13189/cea.2017.050605

Höffken, S., \& Streich, B. (2013). Mobile Participation: Citizen Engagement in Urban Planning via Smartphones. In C. N. Silva (ed), Citizen EParticipation in Urban Governance: Crowdsourcing and Collaborative Creativity (pp. 199-226). https://dx.doi.org/10.4018/978-1-46664169-3.ch011

Hudson-Smith, A., Evans, S., Batty, M., \& Batty, S. (2002, December). Online participation: The Woodberry Down Experience. Centre for Advanced Spatial Analysis: Working Paper Series. London, University College London.

IAP2. (2007). Spectrum of public participation. https://cdn.ymaws.com/www.iap2.org/resource/res mgr/pillars/Spectrum_8.5x11_Print.pdf

Jannack, A., Holmer, T., Stelzle, B., Doll, K., Naumann, F., Wilde, A., \& Noennig, J. (2019). Smart Campus Design - U_CODE Tools Tested for Co-Designing the CJD Learning Campus, Sangerhausen Case Study. In Hands-on Science (ed.). Innovative Education in Science and Technology (pp. 11-20).

Janowitz, M. (2015). Giriş. In R. Park, \& E. W. Burgess, Şehir: Kent Ortamındaki Insan Davranışlarının 
Araştırılması Üzerine Öneriler (p. İstanbul). [City: Recommendations on Researching Human Behaviors in Urban Environment (p. Istanbul)]. Heretik Yayınları:Turkey

Kassen, M. (2018). E-participation actors: understanding roles, connections, partnerships. Knowledge Management Research \& Practice, 18(1), 16-37. https://dx.doi.org/ $10.1080 / 14778238.2018 .1547252$

Kingston, R. (2002). The role of e-government and public participation in the planning process. $X V I$ AESOP Congress. Volos, Greece, July, 10th-14th, 2002.

Klosterman, R. E. (2012). E-Planning: Retrospect and Prospect. International Journal of E-Planning Research, 1(1), 1-4. https://dx.doi.org/10.4018/ijepr.2012010101

Krabina, B. (2016). The E-Participation Ladder Advancing from Unawareness to Impact Participation. in P. Parycek and N. Edelmann (eds.) CeDEM16 Proceedings of the International Conference for E-Democracy and Open Government 2016; 18-20 May; Danube University Krems, Austria. Krems: Edition Donau-Universität Krems, 75-81.

Kubicek, H. (2009). The Potential of E-Participation in Urban Planning: A European Perspective. n: Silva C.N., editor. Handbook of Research on E-Planning: ICTs for Urban Development and Monitoring, IGI Global, 168-194. https://dx.doi.org/10.4018/978-161520-929-3.ch009

Lane, M. B. (2005). Public Participation in Planning: an intellectual history. Australian Geographer, 36(3), 283-299.

https://doi.org/10.1080/00049180500325694

Lefebvre, H. (2016). Şehir Hakkı [Le Droit a la ville]. Istanbul: Sel Yayınc1lık.

Macintosh, A. (2004). Characterizing E-Participation in Policy-Making. 37th Hawaii International Conference on System Sciences, 5-8 Jan. 2004, Big Island, HI, USA. https://doi.org/10.1109/HICSS.2004.1265300

Macintosh, A., \& Whyte, A. (2008). Towards an evaluation framework for eParticipation. Transforming Government: People, Process \& Policy, 2(1), 16-30. https://doi.org/10.1108/17506160810862928
Mansourian, A., Taleai, M., \& Fasihi, A. (2011). A webbased spatial decision support system to enhance public participation in urban planning processes. Journal of Spatial Science, 56(2), 269-282. https://doi.org/10.1080/14498596.2011.623347

Maptionnaire (2011). Maptionnaire. Retrieved April 2020, from Create map-based surveys to get ideas and insights from residents: https://maptionnaire.com

Mueller, J., \& Lu, H. (2017). Designing with citizens: Challenges and evaluation methods for crowdsourced urban layouts. CEUR Workshop Proceedings. 2099(1) 34-39.

Mueller, J., Lu, H., Chirkin, A., Klein, B., \& Schmitt, G. (2018). Citizen Design Science: A strategy for crowd-creative urban design. Cities, 72(1), 181-188. https://doi.org/10.1016/j.cities.2017.08.018

OECD. (2001). Citizens as Partners: Information, Consultation and Public Participation in PolicyMaking. Organization for Economic Cooperation and Development (OECD). https://doi.org/10.1787/9789264195561-en

Poplin, A. (2013). Digital serious game for urban planning: "B3-Design your Marketplace!". Environment and Planning B: Planning and Design, 41(3), 493-511. https://doi.org/10.1068\%2Fb39032

Thiel, S. K. (2017). Let's play urban planner: The use of game elements in public participation platforms. plaNext - next generation planning. 4(1), 58-75. https://doi.org/10.24306/plnxt.2017.04.005

U_CODE. (2019). Pilot test in Sangerhausen, Germany (May/June 2019). Retrieved April 2020, from http://www.u-code.eu/\#

Unlimited Cities DIY. (2017). A free application to transform the city together. Retrieved April 2020, from http://unli-diy.org/EN/

World Urban Campaign. (2016). Unlimited Cities DIY. Retrieved April 2020, from https://www.worldurbancampaign.org/unlimitedcities-diy 
www.ijcua.com

This page is intentionally left blank. 